\title{
Efectos del salario mínimo en el mercado laboral de Honduras, 2007-2011
}

\author{
Marvin J. Martínez, José S. Menjívar' \\ Edwin Medina ${ }^{2}$
}

\section{RESUMEN}

En Honduras, como a nivel de América Latina, el tema del mercado laboral es abordado en forma empírica y carente de objetividad científica. En consecuencia, esta investigación se centra en dar respuesta a esta situación con el fin de analizar los efectos de los ajustes al salario mínimo en la población ocupada económicamente activa del mercado laboral, así como determinar la relación de los ajustes al salario mínimo en la población ocupada del mercado laboral durante el período 2007-2011, estimar la incidencia del salario mínimo en el subempleo visible e invisible de la población económicamente activa del mercado laboral y establecer los niveles de incidencia de dichos ajustes en la población asalariada (público, privado y doméstico).

En este sentido, la investigación se desarrolló basándose en los datos de la encuesta permanente de hogares de propósitos múltiples (EPHPM) del Instituto Nacional de Estadísticas (INE), bajo el uso de modelos de regresión lineal simple, análisis descriptivo y el respaldo de entrevistas a expertos en el tema. Como parte de los resultados encontramos que por cada lempira de aumento al salario mínimo promedio mensual se generan 145 nuevos ocupados, de los cuales 64 pertenecen a cuenta propia, 42 a trabajadores familiares no remunerados y 39 a asalariados públicos, privados y doméstico; considerando que la agricultura, silvicultura, caza y pesca es la actividad económica que mayor número de ocupados genera respecto a su totalidad.

Palabras clave: mercado laboral, salario mínimo, población económicamente activa, población ocupada, subempleo visible e invisible; asalariados públicos, privados y doméstico.

\footnotetext{
${ }^{1}$ Beneficiario de una beca básica de la DICYP. Estudiantes de la Maestría en Metodologías de la Investigación Económica y Social, Facultad de Ciencias Económicas, UNAH: jose.menjivar@unah.edu.hn; marvinjaviers19@hotmail.com

${ }^{2}$ Asesor, Dirección de Investigación Científica y Posgrado, UNAH; edwinhts@yahoo.com
} 


\section{ABSTRACT}

In Honduras as at the regional level in Latin America, this theme of labor market is much addressed empirically lacking computerization of scientific objectivity. Immediately after it, this investigation centres on giving response to this situation by means of his general objectives in order to analyze the effects of the adjustments to the minimum wage in the economically active working population of the labor market, as well as his specific aims, directed in determining the relation of the adjustments to determine the incident to the minimum wage in the working population of the labor market during the period 2007-2011, to estimate the incident of the minimum wage in the visible and invisible underemployment of the economically active population of the labor market and to establish the levels of incident of the above mentioned adjustments in the wage-earning population (I publish, privately and domesticate).

In this respect, the investigation develop on the basis of this information of the survey from the Permanent Survey of Homes of Multiple Intentions (EPHPM) of the National Institute of Statistics (INE), under the model use of linear simple regression, descriptive analysis and the support of interviews to experts in the topic. For your part we find that by every lempira of increase to the average monthly minimum wage there are generated 145 busy new ones, of which 64 belong to own account, 42 to familiar not remunerated workers and 39 to public, private employees and I domesticate. Considering that the agriculture, forestry, hunt and fishing is the activity economic that major number of occupied generates with regard to his totality.

Keywords: labor market, minimum wage, labor force, employed population, visible and invisible underemployment; salaried public, private and domestic.

\section{INTRODUCCIÓN}

El desarrollo de esta investigación tiene como motivo muy importante analizar los efectos del salario mínimo en la población económicamente activa (PEA) del mercado laboral hondureño durante el período 2007-2011. Se espera aportar al estudio de la economía laboral en materia de mercado laboral, brindando nuevos aspectos a considerar por el Gobierno, empresa privada y trabajadores al momento de concretar nuevos acuerdos de negociación, puesto que los recientes estudios enfocados al mercado laboral describen con poca profundidad las incidencias de los ajustes al salario mínimo en dicho mercado. 
Es importante destacar que no siempre con la implementación de políticas laborales, como el salario mínimo, se generan resultados positivos de forma automática para el conjunto de variables que comprende el mercado laboral. La efectividad de estas políticas se refleja a través de la incidencia en los niveles de empleo, en donde durante los últimos años ha existido una fuerte preocupación sobre las consecuencias no deliberadas en el empleo. Producto de ello, diversas corrientes teóricas explican que los ajustes a los salarios mínimos generan una distorsión en los mercados laborales que irremediablemente se traduce en disminución en la demanda de trabajo, generando mayores niveles de desempleo y empleo informal.

Para fines de esta investigación se aborda en gran medida el modelo competitivo del mercado de trabajo de dos sectores: el cubierto por el salario mínimo y el no cubierto, en donde los trabajadores del sector cubierto se desplazan al sector no cubierto por el incremento al salario mínimo. El resultado final de estos dos sectores, producto del incremento del salario mínimo, hace que el empleo global disminuya; también se reduce en el sector cubierto y aumenta generalmente en el sector no cubierto. En este sentido, primero comenzamos con una caracterización del mercado laboral y el análisis de la población ocupada, seguidamente la incidencia en la población con problemas de empleo y por último los efectos en los asalariados.

\section{METODOLOGÍA}

Dada la incertidumbre sobre los efectos de los ajustes al salario mínimo en el mercado laboral, esta investigación se realizó con base al enfoque cuantitativo de carácter explicativo, respecto a las incidencias del mismo, tratando de identificar cuáles han sido sus efectos, ya sean positivos o negativos. Por esta razón, el procedimiento fue enfocado principalmente en el análisis de datos de corte transversal a mayo del período 2007-2011, en conjunto con la aplicación de modelos de regresión lineal simples bajo el método de mínimos cuadrados ordinarios (MCO) en las principales variables del mercado laboral.

Para desarrollar el análisis antes mencionado se contó con las bases de datos de la encuesta permanente de hogares de propósitos múltiples (EPHPM) del Instituto Nacional de Estadísticas (INE), así como cada uno de los decretos ejecutivos aprobados por el Poder Ejecutivo en materia de salario mínimo durante el período de estudio, con el fin de calcular el salario mínimo promedio mensual.

Respecto al contexto de la información, se trabajó con base al total de la población ocupada perteneciente a la PEA a nivel nacional, considerando las categorías de dominio, nivel educativo, rango de edad, sexo, rama de actividad y ocupación, 
haciendo énfasis en que estas categorías son las clasificaciones realizadas por el INE en la EPHPM y que para fines de esta investigación tomaremos esta terminología, sin tener ningún vínculo con el enfoque de la investigación.

Respecto al análisis estadístico, se contó con el programa EViews versión 7, con el cual se trabajó en la elaboración de las regresiones lineales; con el uso previo del paquete de Microsoft Office Excel se elaboraron las bases de datos para un posterior tratamiento en el programa antes mencionado. En este sentido, las variables se trabajaron de forma anual, considerando al salario promedio anual y a las principales variables del mercado laboral obtenidas a mayo de cada año por medio de la EPHPM. Asimismo, se trabajó con un nivel de confianza del $5 \%$ en el desarrollo de cada una de las regresiones simples.

Para darle un mayor respaldo científico a la investigación se realizó una entrevista semiestructurada al especialista en mercado laboral Dr. Henry Rodríguez Corea, coordinador de la Maestría en Metodologías de Investigación Económica y Social de la Universidad Nacional Autónoma de Honduras.

\section{CARACTERIZACIÓN DEL MERCADO LABORAL}

\section{El salario mínimo en Honduras}

El salario mínimo constituye una herramienta de gran importancia en el funcionamiento del mercado laboral y el sistema económico productivo del país, en el cual la empresa privada y trabajadores presentan sus argumentos, posiciones y propuestas orientadas particularmente a obtener su constante maximización de beneficios, en detrimento de los niveles de cohesión entre ambos sectores, originando distorsiones en las principales variables del mercado laboral y de la actividad económica en general (COHEP, 2012).

En el marco de la Ley de Salario Mínimo del 20 enero de 1971, según cifras de la Secretaría del Trabajo y Seguridad Social se han decretado veinte y siete (27) ajustes al salario mínimo, de los cuales; dos (2) se dieron en los años setenta, dos (2) en los ochenta, diez (10) en los noventa y trece (13) del dos mil al dos mil once. Asimismo, hay que mencionar que de su totalidad, doce decretos han sido por la vía del consenso de la comisión tripartita entre empresarios, trabajadores y Gobierno y quince (15) de forma directa por el Poder Ejecutivo, debido a la falta de concertación en las partes interesadas en los procesos de negociación (STSS, 2010).

El repunte en la fijación de salarios mínimos durante la década de los noventa radica en el estricto cumplimiento de la Ley y los elevados niveles de participación y 
apertura de los sectores productivos de la economía nacional, a raíz de la Ley de Transformación del Estado impulsada por el Gobierno central. A partir de ahí surgieron incrementos constantes, en donde en el 2009 se reflejó el mayor crecimiento de $51.5 \%$ en valores constantes correspondiente a 44.97 lempiras diarios, seguido por 1999 con 35.3\% representando 9.06 lempiras diarios y en un tercer lugar 1997 con $35.1 \%$, significando 6.17 lempiras diarios (STSS, 2010).

Por otra parte, según datos de la EPHPM a mayo de cada año en el período de estudio, el 53 \% de la población ocupada en promedio declaró ingresos menores de un salario mínimo con 36 horas de trabajo, seguido por los de 1 a 2 salarios con $19 \%$ y en un último lugar los ocupados de 2 salarios y más con $10 \%$, representando un 83 $\%$ de la totalidad de los que declaran ingresos, existiendo un $17 \%$ que no declararon ningún tipo de ingresos. En cambio, el $52 \%$ del total de asalariados declararon ingresos menores a un salario mínimo y un $41 \%$ obtuvieron ingresos mayores a un salario mínimo.

\section{La población económicamente activa del mercado laboral de Honduras}

De acuerdo a la base de datos de la EPHPM del INE a mayo de 2011, Honduras cuenta con una población de 8,200,795 de habitantes, de los cuales el $48.6 \%$ son hombres y el $51.4 \%$ mujeres, alcanzando los 7.4 años de estudio promedio (AEP), con una PEA de 3,369,919 correspondiente en un $52.4 \%$ al área urbana y un $47.6 \%$ rural.

En este sentido, la PEA en el mercado laboral hondureño se encuentra integrada por los ocupados y desocupados, en donde durante el 2011 se muestra una participación del $96 \%$ y $4 \%$, respectivamente, destacando que los niveles de participación de la población ocupada disminuyó en $1 \%$ a partir de 2010 (97\%). También se muestra que la población en los rangos de 45 a 59 años de edad, son los de mayor participación en el total de la PEA, manteniendo una tendencia de $19 \%$ hasta el 2009, pasando a un $20 \%$ a partir de 2010; en segundo lugar se encuentra los rangos de 19 a 24 años de edad manteniendo una tendencia del $17 \%$ hasta el 2009 , seguido por un $18 \%$ a partir de 2010. Los rangos de 10 a 11 años de edad mantienen una tendencia del $1 \%$ durante el período de estudio, siendo este el de menor participación en su totalidad.

Por otra parte, al analizar la PEA desde el punto de vista de las ramas de actividad económica, la agricultura, silvicultura, caza y pesca en promedio muestran la mayor participación del $35 \%$ durante los últimos cinco años, seguido por el comercio al por mayor y menor, hoteles y restaurantes con $22 \%$ y en un tercer lugar se ubican los 
servicios comunales, sociales y personales con $15 \%$. En cambio, sí lo vemos desde el tipo de ocupación, encontramos que los agricultores, ganaderos y trabajadores agropecuarios poseen la mayor participación en un $34 \%$ en promedio durante el período de estudio, seguido por comerciantes y vendedores con un $15 \%$ y en un tercer lugar los trabajadores de la industria textil, albañilería y mecánica en un $14 \%$.

Gráfico 1. PEA por dominio, 2007-2011

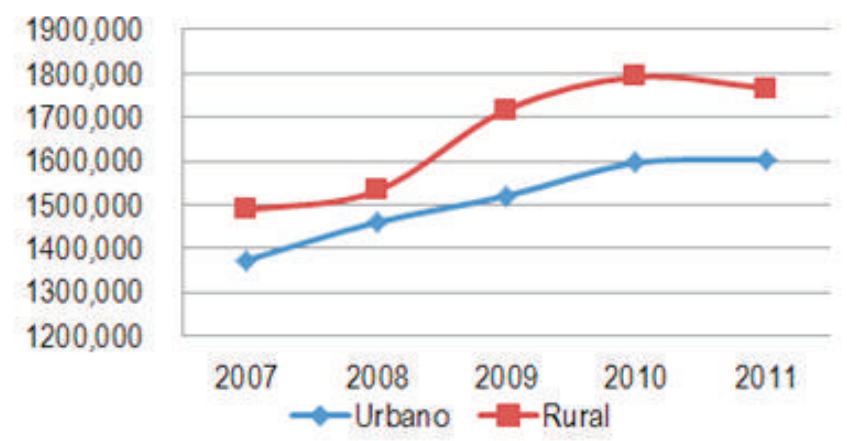

Fuente: Elaboración propia, en base de datos de la EPHPM del INE.

Al analizar la PEA desde el punto de vista de los dominios, se observa que para el 2011 el dominio urbano alcanzó una PEA de 1,603,305 habitantes, de los cuales el $29.1 \%$ se encuentra en el Distrito Central, $16.2 \%$ en San Pedro Sula y el $54.7 \%$ en el resto urbano; es de mencionar que durante el período 2007-2011 existe la tendencia a mantener los mismos niveles de participación.

A partir de 2008, la PEA para el área urbana ha presentado un ritmo de crecimiento decreciente, pasando de $6.26 \%$ en este año, a un $0.48 \%$ para el 2011. Este comportamiento está determinado por los niveles de variación presentado en San Pedro Sula, pasando de un $7.47 \%$ en el 2010 a un $-6.16 \%$ en el 2011 , sumado a las variaciones en 2009 y 2011 para el Distrito Central, con un $1.46 \%$ y $1.26 \%$ respectivamente. En cambio, podemos decir que el resto urbano durante el 2009 mostró un crecimiento del $5.81 \%$, pasando del $4.48 \%$ en el 2008 y, a la vez, manteniendo un ritmo de crecimiento decreciente para los subsiguientes años.

Por otra parte, en el dominio rural en el 2011 se alcanzó una PEA de 1,766,613 habitantes, de los cuales el $26.65 \%$ son mujeres y el $73.35 \%$ hombres. Se destaca que durante el 2008 este dominio presentó el máximo crecimiento del período con un $12.12 \%$ respecto a 2007 , esto sin duda vino a significar el mayor repunte en el total de la PEA durante el período de estudio con un $8.24 \%$ respecto a 2007. 


\section{Población ocupada}

1. Evolución de la población ocupada en el mercado laboral

La población ocupada durante los últimos cinco años ha mantenido un ritmo de crecimiento promedio de $3.9 \%$, en contraste con una disminución del $0.86 \%$ para el año 2011, marcado por un fuerte crecimiento del $8.08 \%$ durante el 2009, debido a la relativa estabilidad económica del país durante los últimos tres años y el apoyo del Estado al sector agrícola a través del bono productivo y la apertura de centros comerciales en el área urbana, como resultado se mostró el máximo crecimiento alcanzado en agricultura, silvicultura, caza y pesca con $17.93 \%$ y el comercio en $13.07 \%$ para este mismo año.

De acuerdo a los datos de la EPHPM, la población ocupada la podemos analizar a través de las categorías de nivel educativo, rango de edad, dominio, sexo, rama de actividad y ocupación. Desde el punto de vista del nivel educativo se identifica que el nivel primario es el que muestra la mayor participación con un $55 \%$ en promedio durante el período 2007-2011, seguido por los de nivel secundario con un $24 \%$ y en un tercer lugar los que no presentan ningún nivel, con $13 \%$ de su totalidad. Asimismo, se muestra que para el 2009 los sin nivel, primaria y secundaria reflejaron los máximos crecimientos del período con $9.79 \%, 9.98 \%$ y $7.67 \%$ respectivamente, en contraste con el nivel superior que mantuvo una disminución del $4.52 \%$.

Gráfico 2. Población ocupada por edad, 2007-2011

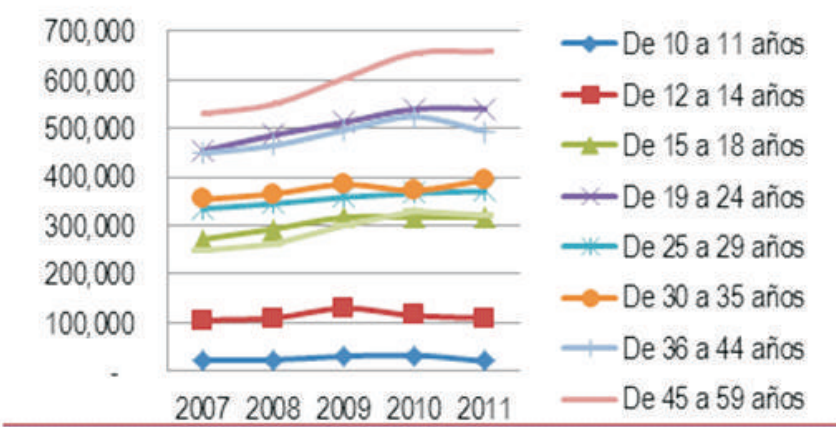

Fuente: elaboración propia con base a datos de la EPHPM del INE.

Por otra parte, se puede decir que la población ocupada con rangos de 45 a 59 años de edad son los de mayor participación, con un $20 \%$ en promedio durante el período 
de estudio; seguido por los de 19 a 24 , con un $17 \%$ y en un tercer lugar los de 36 a 44 , con $16 \%$. De igual modo, durante el 2011 los jóvenes ocupados de 10 a 11 años de edad disminuyeron en $12,343(37 \%)$, seguidos por los de 12 a 14 años de edad con 4,846 (4.24\%), junto a los ocupados de 60 años y más con 9,171 (2.78\%).

En cambio, durante el 2009, surge un comportamiento muy particular al resto, en donde los mayores crecimientos se encuentran en ambos extremos; por un lado tenemos a los de 10 a 11 años con 7,556 (32.36\%), seguido por los de 12 a 14 años con $20,349(18.57 \%)$ y en el otro extremo se encuentran los de 60 años y más con $38,710(14.79 \%)$.

Todo esto debido al fuerte crecimiento de la agricultura, silvicultura, caza y pesca (17.93\%), acompañado por el comercio al por mayor y menor, hoteles y restaurantes (13.07\%), tomando en cuenta que en la siembra de primera de 2009 se otorgó un fuerte apoyo al sector agrícola a través del bono productivo de la Secretaría de Agricultura y Ganadería, sumado al echo que en este sector la principal mano de obra se sustenta por los jóvenes y personas de avanzada edad.

En este sentido, la actividad agrícola resulta ser la de mayor importancia en el mercado laboral hondureño, principalmente para los hombres que viven en el área rural, en donde el $65.05 \%$ de la totalidad de ocupados son hombres y el $34.95 \%$ pertenece a las mujeres, señalando que el 2009 se mantiene como el de mayor crecimiento de $8.74 \%$ y $6.90 \%$, respectivamente. Por otra parte, se identifica que en el 2011 es donde se presentan las

mayores disminuciones; los hombres mostraron un crecimiento de $0.57 \%$ después de un crecimiento de $3.12 \%$ en el 2010 , acompañado por una disminución en las mujeres de $3.77 \%$ luego de un crecimiento de $4.99 \%$ en el mismo período del año anterior.

Este comportamiento radica en que los agricultores, ganaderos y trabajadores agropecuarios disminuyeron en $3.99 \%$, junto a los comerciantes y vendedores con $2.89 \%$, los que constituyen las profesiones de mayor participación en el total de la población ocupada, sumado a los trabadores del área gráfica, química y alimentos con disminuciones de $4.60 \%$ y conductores de transporte con $17.23 \%$, que representan una menor cuantía en su totalidad de ocupados. 
Gráfico 3. Población ocupada por sexo, 2007-2011

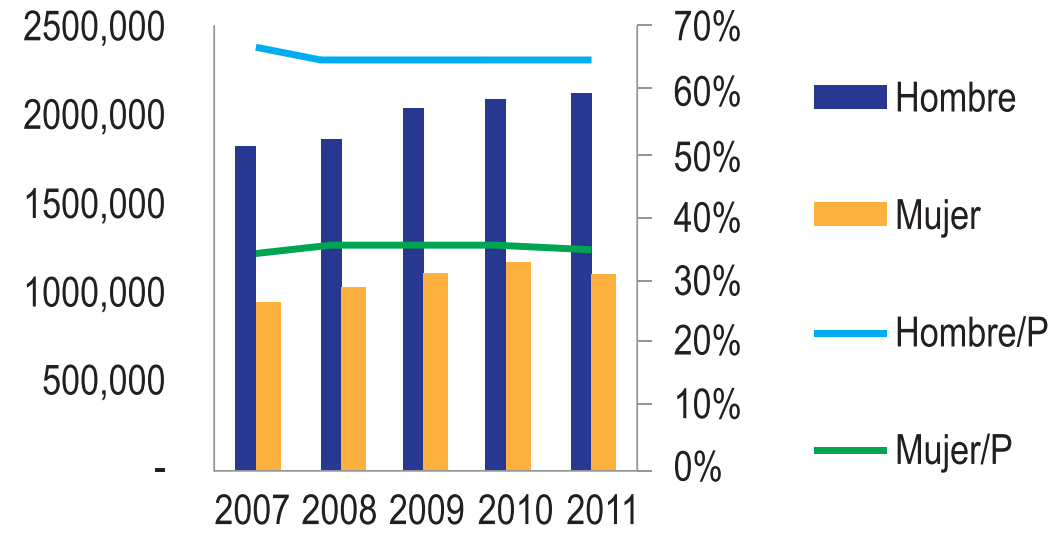

Fuente: elaboración propia con base a datos de la EPHPM del INE.

Relación de los ajustes al salario mínimo en la población ocupada del mercado laboral

En la historia del mercado laboral hondureño se han producido 27 fijaciones de salarios mínimos enmarcadas en la Ley del Salario Mínimo de 1971, en donde a partir del año 2000 se han producido 13 ajustes, de los cuales 5 se dieron durante el 2007-2011; período en el cual la economía nacional alcanzó los niveles de crecimiento más bajos en los últimos diez años, producto de la desaceleración económica internacional, acompañada de los acontecimientos internos de carácter sociopolítico a mediados de 2009, que directa o indirectamente vino a contribuir a la inestabilidad de la actividad económica productiva del país (STSS, 2010).

Según la EPHPM, el comportamiento de la población ocupada a pesar de los acontecimientos acaecidos se ha caracterizado por mantener un fuerte dinamismo, creciendo a un ritmo de $3.9 \%$ anual durante los últimos cinco años, mayor en 1.74 puntos al crecimiento promedio poblacional anual $(2.16 \%)$.

Con el fin de lograr obtener una relación y una contribución del salario mínimo en la población ocupada, se utilizó un modelo lineal simple por el método de mínimos cuadrados (MCC), quedando especificado de la siguiente manera:

Especificación econométrica de forma teórica:

1) OCUPADOS $=\mathrm{C}(1)+\mathrm{C}(2){ }^{*} \mathrm{SALARIO} \_\mathrm{MINIMO}$ 
Donde;

Ocupados: la población total en condición de ocupados

Salario_mínimo: salario mínimo promedio mensual

Estimación econométrica:

\section{2) OCUPADOS $=2419794.76517+145.737367177^{*}$ SALARIO_MINIMO}

Los ajustes al salario mínimo han contribuido significativamente al stock de ocupados, manteniendo una relación directa de tendencia positiva, bajo el supuesto que el número de personas ocupadas está en función del salario mínimo promedio mensual, con un nivel de asociación del $92.56 \%$. El modelo estimado de la población ocupada muestra un coeficiente de determinación del $85.6 \%$ significativamente alto, en donde puede interpretarse como el porcentaje de variabilidad de ocupados ya explicado. En lo que respecta al error estándar, mide la variabilidad o dispersión de los valores observados alrededor de la línea de regresión de ocupados, en este caso se muestran errores estándar moderadamente bajos con tendencia a ser normalizados en los betas individuales.

De esta forma se encontró que por cada lempira promedio de aumento al salario mínimo, se generan 145 nuevos ocupados en su totalidad; de los cuales 64 pertenecen a los cuenta propia, seguido por los trabajadores familiares no remunerados con 42 y 39 correspondientes a los asalariados públicos, privados y doméstico con 5,32 y 2 respectivamente.

Tabla 1. Contribución del salario mínimo a la población ocupada, 2007-2011

\begin{tabular}{|l|c|c|c|c|}
\hline \multicolumn{1}{|c|}{ Variable } & Coefficient & Std. Error & t- Statistic & Prob. \\
\hline C & 2419795 & 156195.3 & 15.49211 & 0.0006 \\
\hline SALARIO_MINIMO & 145.7374 & 34.39881 & 4.236698 & 0.0241 \\
\hline R-squared & 0.856799 & Mean dependent var & & 3058049 \\
\hline Adjusted R-squared & 0.809066 & S.D. dependent var & & 211106.6 \\
\hline S.E. of regression & 92245.23 & Akaike info criterion & & 25.99146 \\
\hline Sum squared resid & $2.55 E+10$ & Schawarz criterion & & 25.83524 \\
\hline Log likelihood & -62.97866 & Hannan- Quinn criter & & 25.57217 \\
\hline F-statistic & 17.94961 & Durbin- Wats on stat & & 2.201559 \\
\hline Prob(F-static) & 0.024071 & & & \\
\hline
\end{tabular}

Fuente: elaboración propia con datos de la EPHPM.

De acuerdo a los test individuales en el modelo especificado de la población ocupada, se parte de la hipótesis nula que los betas individuales son iguales a cero, 
por lo tanto, de acuerdo a las probabilidades arrojadas en la estimación se rechaza la hipótesis nula y se acepta la hipótesis alternativa que los betas son diferentes a cero. En este sentido, se dice que los betas individuales son estadísticamente significativos, manteniendo un nivel de confianza del $5 \%$.

Desde el punto de vista del análisis ANOVA, la probabilidad que los betas en su conjunto sean iguales a cero es P: 0.02; por lo tanto, se rechaza la hipótesis nula, aceptando que el modelo globalmente es estadísticamente significativo utilizando un $5 \%$ de nivel de significancia.

Según una entrevista realizada al Dr. Henry Rodríguez (2012), realmente no se generan 145 nuevos ocupados, sino más bien son 145 nuevas personas con deseo de trabajar, principalmente aquellos trabajadores con baja formación profesional y experiencia que se ven motivados por dichos aumentos al salario mínimo. En esta misma dirección menciona que esta relación directa contradice la lógica económica de la oferta y la demanda del mercado de trabajo, pero que realmente el comportamiento radica en el momento en que se realizó las encuestas, considerando que la mayor cantidad de ocupados se encuentra en el sector agrícola y este es un sector que genera empleo de forma estacional, principalmente en la siembra de primera en los meses de abril, mayo y junio y es el momento en el que se realizan las encuestas de hogares.

La mayor incidencia de los ajustes al salario mínimo recae en los ocupados pertenecientes al sector informal, normalmente conocidos como cuenta propia y trabajadores familiares no remunerados. En este sentido, se identifica que los cuenta propia marcaron un ritmo de crecimiento promedio de $3.68 \%$, a excepción de 2011 que presentaron una caída de $8.07 \%$, acompañado por un fuerte crecimiento en 2009 de $12.17 \%$. Este comportamiento se debe al máximo incremento de 51.55 $\%$ (L.52.49 diarios) al salario mínimo, lo que produjo que los asalariados mostraran una participación del $46 \%$ del total de ocupados en el 2009 , luego de haber obtenido un $48 \%$ en el 2008 , lo que se convirtió en un $42 \%$ de participación en los cuenta propia en el 2009 después de un $40 \%$ en el mismo lapso del año anterior. También, los trabajadores familiares no remunerados alcanzaron un crecimiento promedio de $9.64 \%$, logrando alcanzar un máximo crecimiento de $13.53 \%$ para el 2009 y obteniendo el menor crecimiento de $3.38 \%$ durante el 2011.

El comportamiento de la población ocupada es atípica en 2009, en donde logra alcanzar el máximo crecimiento del período con $8.08 \%(234,488)$, de los cuales el $81 \%$ corresponde a los cuenta propia y trabajadores familiares no remunerados con $60.94 \%(142,890)$ y $19.72 \%(46,242)$ respectivamente, seguido por un $19.34 \%$ correspondiente a los ocupados asalariados. Por otra parte, cabe mencionar que 
durante este mismo año la agricultura, silvicultura, caza y pesca generaron 176,605 nuevos ocupados, seguido por el comercio al por mayor y menor, hoteles y restaurantes con 80,098 nuevos ocupados, logrando generar un total de 256,703 en ambas actividades; este resultado no se refleja en la totalidad de ocupados debido a la incidencia negativa que produjo el ajuste al salario mínimo en la industria manufacturera con una disminución del $3.52 \%(14,996)$, establecimientos financieros, seguros, bienes inmuebles y servicios con $7.88 \%(8,161)$ y los servicios comunales, sociales y personales con una disminución del $2.69 \%$ (12,241 empleos).

La incidencia de los ajustes al salario mínimo en los ocupados asalariados no se ve reflejada en significativas disminuciones en su stock durante el período de estudio, a excepción del 2010 con una disminución de $1.92 \%$. Este comportamiento radica en el constante crecimiento vegetativo de la población en edad de trabajar y de la actividad económica en general como parte de su propio circulo vicioso, en tal sentido, la incidencia que provocan los ajustes es retardar el crecimiento acelerado de los ocupados asalariados, generando un efecto traslado a los ocupados no asalariados o mejor dicho a aquellos trabajadores no cubiertos por el salario mínimo (cuenta propia, trabajadores familiares no remunerados), además con este efecto se contribuye en gran medida aumentando los niveles de desempleo abierto (total PEA menos total ocupados), la dinamización del número de ocupados con problemas de empleo (subempleo visible e invisible) y al mayor incumplimiento del salario mínimo por parte de los empleadores.

En síntesis, la población ocupada siempre va presentar una tendencia de crecimiento positivo, principalmente en el área rural, indistintamente de la magnitud de los ajustes al salario mínimo debido al efecto traslado de los ocupados asalariados a los informales (cuenta propia, trabajadores familiares no remunerados) y la fuerte dependencia de la agricultura, silvicultura, caza y pesca en el total de ocupados, considerando también que esta actividad está en función de la dinámica de los precios de los productos agropecuarios en el mercado nacional e internacional, desastres naturales y la gestión de políticas públicas.

\section{Población con problemas de empleo}

1. Incidencia del salario mínimo en el subempleo visible de la población ocupada

Para fines de esta investigación se considera como subempleado visible a toda aquella persona que ha trabajado por un tiempo menor al considerado normal en la actividad específica. Para estar dentro de esta clasificación es necesario que la

\section{8}


persona desee trabajar una jornada más larga, aun cuando no le haya sido posible. Este indicador es caracterizado por su alta complejidad de medición, relacionado en definir el tiempo normal de la actividad, pero es el de mayor utilización en los países de la región (CEPAL, 1997).

Según datos de la EPHPM a mayo de 2011, en Honduras existe un total de $1,854,033$ personas con problemas de empleo (desocupados, subempleo visible e invisible, potencialmente activos y desalentados), caracterizados por presentar un crecimiento promedio de $10.55 \%$ durante el período 2007-2011, mostrando una fuerte disminución para el 2008 de $10.01 \%$ (128,770), acompañado de un significativo aumento de $29.89 \%(345,855)$ en el 2009. Debido al máximo crecimiento a los ajustes al salario mínimo en valores constantes durante los últimos 42 años.

Por otra parte, hay que mencionar que el subempleo visible para el 2011 representa el $18.01 \%$ del total de personas con problemas de empleo, caracterizados principalmente por mantener un nivel académico de 7.3 años de estudio promedio, perteneciendo un $52.76 \%(176,202)$ de su totalidad al dominio rural y un $47.24 \%$ $(157,767)$ al urbano.

Con el fin de lograr obtener una relación y una contribución del salario mínimo en la población en condición de subempleo visible, se utilizó un modelo lineal simple por el método de mínimos cuadrados (MCC), quedando especificado de la siguiente manera:

Especificación econométrica de forma teórica:

1) SUBEMPLEO_VISIBLE $=C(1)+C(2)^{*}$ SALARIO_MINIMO

Donde;

Subempleo visible: la población total en condición de subempleo visible

Salario mínimo: salario mínimo promedio mensual

Estimación econométrica:

2) SUBEMPLEO_VISIBLE $=-67296.0410755+$ 59.6584431873*SALARIO_MINIMO

Los ajustes al salario mínimo han contribuido moderadamente al stock de subempleados visibles, manteniendo una relación directa de tendencia positiva, bajo el supuesto que el total de personas subempleadas visibles están en función 
del salario mínimo promedio mensual, con un nivel de asociación del $84.31 \%$ sumado a un $71.09 \%$ de explicación y un $95 \%$ de nivel de confianza. Al respecto, se encontró que por cada lempira de aumento al salario mínimo promedio mensual, se contribuye en la generación de 59 nuevos subempleos visibles, de los cuales 31 pertenecen al área rural y 28 al área urbana, recayendo principalmente en los rangos de edades de 10 a 29 años con 27 , seguido por los de 30 a 49 años de edad con 22 y por último a los de 50 años en adelante con 10 subempleos.

Tabla 2. Contribución del salario mínimo a la población en condición de subempleo visible, 2007-2011

\begin{tabular}{|l|c|c|c|c|}
\hline \multicolumn{1}{|c|}{ Variable } & Coefficient & \multicolumn{1}{c|}{ Std. Error } & t- Statistic & Prob. \\
\hline \multicolumn{1}{c}{ C } & -67296.04 & 99732.97 & -0.674762 & 0.5482 \\
\hline SALARIO_MINIMO & 59.65844 & 21.96414 & 2.716175 & 0.0728 \\
\hline R-squared & 0.710916 & Mean dependent var & & 193977.2 \\
\hline Adjusted R-squared & 0.614555 & S.D. dependent var & & 94870.96 \\
\hline S.E. of regression & 58899.91 & Akaike info criterion & & 25.09424 \\
\hline Sum squared resid & $1.04 E+10$ & Schawarz criterion & & 24.93802 \\
\hline Log likelihood & -60.7356 & Hannan- Quinn criter & & 24.67495 \\
\hline F-statistic & 7.377607 & Durbin- Wats on stat & & 1.827514 \\
\hline Prob(F-static) & 0.072784 & & & \\
\hline
\end{tabular}

Fuente: elaboración propia con datos de la EPHPM.

De acuerdo a los test individuales en el modelo especificado de la población en condición de subempleo visible, se parte de la hipótesis nula que los betas individuales son iguales a cero, por lo tanto, de acuerdo a las probabilidades arrojadas en la estimación se rechaza la hipótesis nula y se acepta la hipótesis alternativa que el beta del salario mínimo es diferente a cero, caso contrario a la constante. En este sentido, se dice que el beta individual del salario mínimo es estadísticamente significativo, manteniendo un nivel de confianza del $10 \%$.

Desde el punto de vista del análisis ANOVA, la probabilidad que los betas en su conjunto sean iguales a cero es $\mathrm{P}: 0.07$, por lo tanto, se rechaza la hipótesis nula, aceptando que el modelo globalmente es estadísticamente significativo utilizando un $10 \%$ de nivel de significancia.

En Honduras las personas en situación de subempleo visible se han caracterizado por mostrar un crecimiento acelerado, tal es el caso del período 2007-2011, en el cual se muestra una tasa promedio del $29.77 \%$; respaldado en gran medida por el significativo aumento del $86.07 \%(115,416)$ en el 2010 . Caso contrario sucede en el 2008, en donde se muestra una disminución del $22.96 \%(32,720)$. Su totalidad 
representa en promedio el $15 \%$ de la población con problemas de empleo y un $7 \%$ respecto a los ocupados. Por otra parte, si lo vemos desde el punto de vista del dominio, encontramos que el 54 \% pertenece al área rural y un $46 \%$ para el área urbana. El primero se caracteriza por presentar el mayor crecimiento en el período con $102.68 \%(72,139)$ en el 2010 , acompañado por una significativa disminución de $34.02 \%(28,149)$ en el 2008.

La incidencia de los ajustes al salario mínimo, vía subempleo visible, recae principalmente en la población joven en las edades de 18 a 24 años, con una participación promedio de $18 \%$, seguido por los de 15 a 18 y 25 a 29 años con $12 \%$ y en un tercer lugar los de 30 a 34 junto a los de 35 a 39 años con $11 \%$. Este comportamiento está asociado en gran medida a los bajos niveles de formación profesional, escasa experiencia cualificada, la falta de ingresos y la adquisición de compromisos de carácter familiar. En cambio, la población de 50 años en adelante en su mayoría cuenta con una vasta experiencia o algún tipo de formación profesional, acompañados de una relativa estabilidad en el hogar. Por su parte, los jóvenes menores de 15 años presentan una baja participación debido a que en su mayoría aún cuentan con el respaldo de sus padres o se encuentran en proceso de formación estudiantil de carácter formal e informal.

En otro sentido, se puede decir que la agricultura, silvicultura, casa y pesca es la actividad económica que mayores niveles de subempleo visible ha mostrado durante el período en análisis, con una participación promedio del $33 \%$, seguido por el comercio al por mayor y menor, hoteles y restaurantes junto al subsector de servicios comunales, sociales y personales con $22 \%$ e industria manufacturera con $13 \%$ de su totalidad. Asimismo, se identifica que las ocupaciones que más se destacan en este indicador son los agricultores, ganaderos y trabajadores agropecuarios con $32 \%$, ocupación de los servicios con $16 \%$ y comerciantes y vendedores con $15 \%$.

En síntesis, los ajustes al salario mínimo inciden significativamente en el aumento del stock de subempleados visibles, principalmente en el dominio rural y en la población de 15 a 39 años de edad, debido a la fuerte dependencia de la agricultura, silvicultura, caza y pesca, comercio por mayor y menor, hoteles y restaurantes y servicios comunales, sociales y personales, junto a los altos niveles de pobreza que motivan en la adquisición de cierto tipo de trabajo con el fin de satisfacer las necesidades básicas insatisfechas del hogar. Al respecto, es posible decir que los altos niveles de población con problemas de empleo radica en la incidencia de los ajustes al salario mínimo, formación profesional y el stock de pobreza. 
2. Incidencia del salario mínimo en el subempleo invisible de la población ocupada

Para propósitos de esta investigación entendemos que el subempleo invisible es un indicador que revela la mala asignación del recurso humano, el bajo ingreso, la subutilización de personal y la baja productividad. Destacando que el subempleo invisible se encuentra estrechamente relacionado a los niveles de ingreso asignados como remuneración por debajo al valor de su productividad o al capital humano que realiza tareas que requieren una preparación inferior a la suya (CEPAL, 1997).

El subempleo invisible a mayo de 2011, según la EPHPM, representa el $63.16 \%$ de un total de 1,854,033 personas con problemas de empleo en Honduras, caracterizados por mantener un nivel académico de 6.2 años de estudio promedio, perteneciendo un $60.68 \%(710,549)$ al dominio rural y un $39.32 \%(460,426)$ al urbano.

Con el fin de lograr obtener una relación y una contribución del salario mínimo en la población en condición de subempleo invisible se utilizó un modelo lineal simple por el método de mínimos cuadrados (MCC), quedando especificado de la siguiente manera:

Especificación econométrica de forma teórica:

1) SUBEMPLEO_INVISIBLE $=C(1)+C(2)^{*}$ SALARIO_MINIMO Donde

Subempleo invisible: la población total en condición de subempleo invisible Salario mínimo: salario mínimo promedio mensual

Estimación econométrica:

2) SUBEMPLEO_INVISIBLE $=599196.484206+$ 96.9294713491*SALARIO_MINIMO

Los ajustes al salario mínimo muestran una contribución significativa al stock de subempleados invisibles, arrojando una relación directa de tendencia positiva, bajo el supuesto que el total de población en condición de subempleo invisible están en función del salario mínimo promedio mensual, con un nivel de asociación del 92.20 $\%$. El modelo estimado de la población en condición de subempleo invisible muestra un coeficiente de determinación del $85.01 \%$, significativamente alto, en donde puede interpretarse como el porcentaje de variabilidad de subempleo 
invisible explicado. En lo que respecta al error estándar, mide la variabilidad 0 dispersión de los valores observados alrededor de la línea de regresión de ocupados, en este caso se muestran errores estándar moderadamente bajos con tendencia a ser normalizados en los betas individuales.

Tabla 3. Contribución del salario mínimo a la población en condición de subempleo invisible, 2007-2011

\begin{tabular}{|l|c|c|c|c|}
\hline \multicolumn{1}{c|}{ Variable } & Coefficient & \multicolumn{1}{c|}{ Std. Error } & t- Statistic & Prob. \\
\hline \multicolumn{1}{c}{ C } & 599196.5 & 106685.8 & 5.616459 & 0.0112 \\
\hline SALARIO_MINIMO & 96.92947 & 23.49536 & 4.125473 & 0.0258 \\
\hline R-squared & 0.850146 & Mean dependent var & & 1023698 \\
\hline Adjusted R-squared & 0.800195 & S.D. dependent var & & 140954.7 \\
\hline S.E. of regression & 63006.1 & Akaike info criterion & & 25.22903 \\
Sum squared resid & $1.19 E+10$ & Schawarz criterion & & 25.0728 \\
\hline Log likelihood & -61.07256 & Hannan- Quinn criter & & 24.80973 \\
\hline F-statistic & 17.01953 & Durbin- Wats on stat & & 3.218175 \\
\hline Prob(F-static) & 0.025825 & & & \\
\hline
\end{tabular}

Fuente: elaboración propia con datos de la EPHPM.

De acuerdo a los test individuales en el modelo especificado de la población en condición de subempleo invisible, se parte de la hipótesis nula que los betas individuales son iguales a cero, por lo tanto de acuerdo a las probabilidades arrojadas en la estimación se rechaza la hipótesis nula y se acepta la hipótesis alternativa que los betas son diferentes a cero. En este sentido, se dice que los betas individuales son estadísticamente significativos, manteniendo un nivel de confianza del $5 \%$.

Desde el punto de vista del análisis ANOVA, la probabilidad que los betas en su conjunto sean iguales a cero es $\mathrm{P}: 0.02$, por lo tanto, se rechaza la hipótesis nula, aceptando que el modelo globalmente es estadísticamente significativo utilizando un $5 \%$ de nivel de significancia.

Por su parte, se identifica que por cada lempira de aumento al salario mínimo promedio mensual se contribuye en la generación de 97 nuevos subempleos invisibles, de los cuales 66 pertenecen al dominio urbano y 31 al rural, recayendo principalmente en los rangos de edades de 19 a 29 años con 36 , seguido por los de 30 a 44 años de edad con 34 y en un tercer lugar los de 45 a 59 con 25.

Por lo general, el subempleo invisible muestra un comportamiento contrario a lo ocurrido en el subempleo visible, en donde muestra que su mayor participación recae en el área rural con 54 \% promedio de su totalidad; en cambio en el invisible, 
los mayores niveles se encuentran posicionados en el área urbana con $63 \%$ en promedio, junto a un $37 \%$ para el área rural y, a la vez, caracterizado por mostrar un significativo crecimiento del $34.8 \%(291,177)$ en el 2009, seguido por el 2011 con $10.29 \%(109,272)$, acompañado de una relativa mejoría en cuanto a las disminuciones presentadas durante los años 2008 y 2011 con $-9.16 \%(84,397)$ y $5.87 \%(66,233)$, respectivamente. A lo interno del área urbana se encuentra que el resto urbano es el de mayor participación con $64 \%$, seguido por el Distrito Central con $22 \%$ y un $14 \%$ en San Pedro Sula.

La fuerte incidencia del subempleo invisible en el área urbana recae principalmente en el resto urbano y Distrito Central, lo que radica en el tipo de actividad económica, la edad y la ocupación en la cual se desempeñan. Sobre esto, se señala que en la agricultura, silvicultura, caza y pesca es donde se encuentran los mayores niveles de participación con $49 \%$ en promedio de su totalidad, seguido por el comercio al por mayor y menor, hoteles y restaurantes con $19 \%$ y en un tercer lugar la industria manufacturera junto a los servicios comunales, sociales y personales con $10 \%$ cada uno. Por otra parte, la agricultura, silvicultura, caza y pesca ha venido mostrando un comportamiento hacia la baja, notablemente en el 2008 con una disminución del $18.28 \%(96,494)$, excepto en el 2009 , que experimento un significativo aumento del $24.7 \%(106,592)$ de su totalidad, contribuyendo en gran medida al stock de población con problemas de empleo a raíz del fuerte repunte en los ajustes al salario mínimo, acompañado del declive en la actividad económica, producto de la crisis económica internacional.

En síntesis, los altos niveles de subempleo invisible son movidos por el limitado número de trabajadores amparado por un seguro de protección social. Frente a este tipo de condición, muy poca población puede estar desempleada durante un largo tiempo. La necesidad de cubrir sus necesidades en el hogar obliga a desempeñarse en cierta actividad económica, por precaria que sea. A pesar de que también puedan estar buscando otro empleo o trabajo adicional, no se les contará como desempleados (CEPAL,1997). A raíz de ello la agricultura, silvicultura, caza y pesca, comercio por mayor y menor, hoteles y restaurantes y la industria manufacturera junto a los servicios comunales, sociales y personales, siguen siendo los sectores de la economía que mayor empleo generan. Igualmente cabe mencionar que el dominio urbano es el que presenta mayor dinamismo, soportando en gran medida la incidencia de los ajustes al salario mínimo.

\section{Población asalariada}

Para el análisis de los efectos de los ajustes al salario mínimo en los asalariados públicos, privados y domésticos, se parte prescindiendo del supuesto que de que 
todos los trabajadores están cubiertos por el salario mínimo, en donde consideramos que existen dos sectores: el cubierto por el salario mínimo y el no cubierto. Este tipo de análisis lleva a vislumbrar en dónde recaen dichos efectos, debido a que en la economía hondureña existen muchos subsectores que no están cubiertos por el salario mínimo.

La población asalariada a mayo de 2011, según la EPHPM, representa el $45.69 \%$ $(1,474,119)$ del total de ocupados $(3,226,135)$, mostrando un ritmo de crecimiento promedio del $2.79 \%$ durante el período de estudio. De igual forma, se identifica que hay un total de 205,982 asalariados públicos que representan el $13.97 \%$ del total de asalariados.

Con el fin de lograr obtener una relación y una contribución del salario mínimo en el total de asalariados, se utilizó un modelo lineal simple por el método de mínimos cuadrados (MCC), quedando especificado de la siguiente manera:

Especificación econométrica de forma teórica:

1) TOTAL_ASALARIADOS $=C(1)+C(2)^{*} S A L A R I O \_M I N I M O$ Donde;

Total asalariados: la población total en condición de asalariados

Salario mínimo: salario mínimo promedio mensual

Estimación econométrica:

2) TOTAL_ASALARIADOS $=1233888.64886+$ 38.5701065151*SALARIO_MINIMO

En este sentido, los ajustes al salario mínimo muestran una contribución significativa al stock de asalariados, arrojando una relación directa de tendencia positiva, bajo el supuesto que el total de la población en condición de asalariado está en función del salario mínimo promedio mensual. El modelo estimado de la población en condición de asalariados muestra un coeficiente de determinación del $84.71 \%$ significativamente alto, en donde puede interpretarse como el porcentaje de variabilidad de asalariados explicado. En lo que respecta al error estándar nos mide la variabilidad o dispersión de los valores observados alrededor de la línea de regresión de ocupados, en este caso se muestran errores estándar moderadamente bajos con tendencia a ser normalizados en los betas individuales. 
Tabla 4. Contribución del salario mínimo a la población en condición de subempleo invisible, 2007-2011

\begin{tabular}{|l|c|c|c|c|}
\hline \multicolumn{1}{|c|}{ Variable } & Coefficient & Std. Error & t- Statistic & Prob. \\
\hline C & 1233889 & 42956.75 & 28.72397 & 0.0001 \\
\hline SALARIO_MINIMO & 38.57011 & 9.460342 & 4.077031 & 0.0266 \\
\hline R-squared & 0.847112 & Mean dependent var & & 1402806 \\
\hline Adjusted R-squared & 0.796149 & S.D. dependent var & & 56188.96 \\
\hline S.E. of regression & 25369.23 & Akaike info criterion & & 23.40964 \\
\hline Sum squared resid & $1.93 E+09$ & Schawarz criterion & & 23.25341 \\
\hline Log likelihood & -56.52409 & Hannan- Quinn criter & & 22.99034 \\
\hline F-statistic & 16.62218 & Durbin- Wats on stat & & 2.397556 \\
\hline Prob(F-static) & 0.026641 & & & \\
\hline
\end{tabular}

Fuente: elaboración propia con datos de la EPHPM.

De acuerdo a los test individuales en el modelo especificado de la población en condición de asalariados, se parte de la hipótesis nula que los betas individuales son iguales a cero, por lo tanto, de acuerdo a las probabilidades arrojadas en la estimación se rechaza la hipótesis nula y se acepta la hipótesis alternativa que los betas son diferentes a cero. En este sentido, se dice que los betas individuales son estadísticamente significativos, manteniendo un nivel de confianza del $5 \%$.

Desde el punto de vista del análisis ANOVA, la probabilidad que los betas en su conjunto sean iguales a cero es P: 0.02 , por lo tanto, se rechaza la hipótesis nula, aceptando que el modelo globalmente es estadísticamente significativo utilizando un $5 \%$ de nivel de significancia.

En términos generales podríamos decir que los asalariados públicos han presentado un crecimiento promedio de $3.52 \%$ durante el período, respaldado en gran medida por el significativo crecimiento en el 2008 de $9.33 \%$, en contraste con una relativa disminución del $3.76 \%$ en el 2009. Este último comportamiento realmente refleja la lógica de la ley de la oferta y la demanda del mercado laboral, que cuando se aumenta el salario mínimo disminuyen las cantidades de empleo.

Desde el punto de vista de los dominios encontramos que en promedio el $79.46 \%$ de los asalariados públicos pertenecen al dominio urbano, seguido por un $20.54 \%$ correspondiente al rural. Por su parte se puede decir que la mayor incidencia en los niveles de asalariados públicos recae en el dominio urbano durante el 2009, año en el que se produjo el mayor crecimiento en los ajustes al salario mínimo en términos de valores constantes con un $51.55 \%$. Este comportamiento radicó principalmente en el paro de contrataciones y el despido de personal supernumerario en las oficinas del Estado, con el único fin de salvaguardar las finanzas públicas y mantenerse dentro del margen de los indicadores de la política fiscal. 
Los asalariados públicos con nivel educativo secundario son los de mayor incidencia en términos relativos, seguidos por el nivel superior y primario.

Desde el punto de vista de la actividad económica encontramos que en promedio el $97.49 \%$ del total de asalariados públicos pertenecen a las actividades de servicios comunales, sociales y personales con un $89.91 \%$; electricidad, gas y agua con 3.97 $\%$ y transporte almacenamiento y comunicaciones con $3.61 \%$. También, por el lado de las ocupaciones se identifica que el $82.88 \%$ de los asalariados se encuentra en los profesionales técnicos con $60.59 \%$, ocupación de los servicios con $12.48 \%$ y un $9.81 \%$ de directores, gerentes y administradores generales. En este comportamiento se refleja la mayor participación de los asalariados con nivel de educación secundaria, pertenecientes al área urbana.

En el análisis de la incidencia de los ajustes al salario mínimo en los asalariados privados al igual que los asalariados públicos, partimos de la existencia de los sectores cubiertos y no cubiertos por el salario mínimo. Considerando que este enfoque contribuye en tener una mejor claridad en las incidencias debido a la existencia de muchas actividades económicas que no están cubiertos por el salario mínimo, en este mismo sentido se incluye el elemento del incumplimiento legal del salario mínimo por parte de las empresas.

Los asalariados privados a mayo de 2011, según la EPHPM, representan el $80.28 \%$ $(1,183,426)$ del total de asalariados $(1,474,119)$, mostrando un ritmo de crecimiento promedio del $2.6 \%$ durante el período de estudio. Su mayor crecimiento es alcanzado en el 2009 con un $5.74 \%(63,131)$, en contraste con una relativa disminución del $3.43 \%(39,912)$ en el 2010.

El mercado laboral hondureño se encuentra con una gran dependencia del desempeño de la actividad económica del sector privado, en donde durante los últimos cinco años presentó un promedio del $36.95 \%$ de participación respecto al total de ocupados. También encontramos que el $54.86 \%$ de su totalidad pertenecen al dominio urbano y un $45.14 \%$ al rural. A lo interno del área urbana el resto urbano es el de mayor participación con $49.75 \%$, seguido por el Distrito Central con $28.78 \%$ y en último lugar San Pedro Sula con $21.47 \%$. En esta misma se refleja que el dominio urbano mostró un ritmo de crecimiento promedio de $1.66 \%$, presentando su mayor crecimiento en el año 2011 con $3.4 \%$ (21,090); en cambio, en el dominio rural se mostró un significativo crecimiento del $3.93 \%$ mayor en $2.27 \%$ al del urbano, respaldado principalmente por el fuerte repunte en el 2009 con $12.93 \%(62,746)$, en contraste con una disminución del $8.45 \%(46,319)$ en el 2010. 
Según el nivel educativo, en promedio el $51.4 \%$ de los asalariados privados pertenecen al nivel primario, seguido por los de secundaria con $28.7 \%$ y un $9.9 \%$ sin nivel. Igualmente se identifica que el $55.98 \%$ de su totalidad se encuentran en las edades de 19 a 35 años, estando conformadas por un $25.94 \%$ en los rangos de 19 a 24 , seguido por los de 25 a 29 con $15.94 \%$ y en tercer lugar los de 30 a 35 con $14.10 \%$.

Por otra parte, los stock de asalariados privados muestran su mayor dependencia en las actividades de la agricultura, silvicultura, caza y pesca con $29.01 \%$ de su totalidad, seguido por la industria manufacturera con $20.24 \%$ y en un tercer lugar el comercio por mayor y menor, hoteles y restaurantes con el $19.48 \%$ sumando un total de $68.72 \%$ en promedio de participación en el total de asalariados del sector privado. Asimismo, se identifica que en promedio, la ocupación de agricultores, ganaderos y trabajadores agropecuarios son de mayor contribución con el $26.35 \%$, seguido por los trabajadores de la industria textil, albañilería y mecánica con 23.01 $\%$ y un $10.7 \%$ en las ocupaciones de servicios. El comportamiento en la actividad agrícola, silvicultura, caza y pesca durante el período, principalmente en el 2009, se debe al acompañamiento del Estado a los pequeños y medianos productores a través del bono productivo en la producción de maíz, frijoles y sorgo, sumado al mejoramiento del precio del café que incentivó a la expansión de área de cultivo, tomando en cuenta la aseveración del Dr. Henry Rodríguez, en que la estacionalidad en la cosecha de primera influye en los altos niveles de asalariados privados en las encuestas realizadas a mayo de cada año, lo cual lleva a la contradicción de la lógica económica del mercado laboral.

En este mismo sentido, se identifica que durante el período 2007-2011 en promedio existe un $56.30 \%$ de incumplimiento en el salario mínimo por parte del sector privado, debido a la fuerte dependencia de la actividad agrícola estacionaria en el área rural; dicho de otra forma, esto representa el total de asalariados que declaran ingresos menores a un salario mínimo.

Los asalariados domésticos a mayo de 2011, según la EPHPM, representan el 5.75 $\%(84,711)$ del total de asalariados $(1,474,119)$, mostrando un ritmo de crecimiento promedio del $5.15 \%$ durante el período de estudio. Su mayor crecimiento es alcanzado en el 2008 con un $23.96 \%(17,126)$, en contraste con una fuerte disminución del-11.69\% (10,363) en el 2009.

En términos generales podríamos decir que el $65.76 \%$ de los asalariados domésticos se encuentran en el área urbana, acompañado de un $34.24 \%$ en área rural. En este mismo sentido se identifica que los asalariados domésticos del área 
urbana muestran un ritmo de crecimiento promedio de $3.41 \%$, respaldado en gran medida por el crecimiento significativo del $27.89 \%(13,174)$ en el 2008.

Según el nivel educativo encontramos que en promedio el $63.90 \%$ de los asalariados domésticos pertenecen al nivel primario, seguido por los de secundaria con $24.02 \%$ y un $10.75 \%$ sin nivel. Se identifica que en promedio el $23.01 \%$ de su totalidad se encuentran en las edades de 19 a 24 años, seguido por los rangos de 15 a 18 con $18.81 \%$ y en tercer lugar los de 36 a 39 con $14 \%$.

Por otra parte, el stock de asalariados domésticos muestra su mayor dependencia en la actividad de servicios comunales, sociales y personales con un $99 \%$. Asimismo, se identifica que en promedio el $92.62 \%$ son mujeres con un ritmo de crecimiento del $5.74 \%$, respaldado en gran medida por el aumento significativo en el 2008 con $22.07 \%$, contrario a una disminución de 11.78 en el 2009, en cambio los hombres solamente representan una participación del $7.32 \%$ con una disminución promedio de $1.17 \%$ durante el período, mencionando que solamente en el 2008 se mostró un crecimiento del $47.53 \%$.

\section{CONCLUSIONES}

La población ocupada muestra una relación directa de tendencia positiva respecto a los ajustes al salario mínimo, respaldado en gran medida por los efectos traslados del sector cubierto por el salario mínimo hacia el sector no cubierto, la estacionalidad de los empleos en el área rural y la apertura de centros comerciales en el área urbana, recayendo principalmente en los ocupados cuenta propia y trabajadores familiares no remunerados en la actividad de agricultura, silvicultura, caza y pesca, comercio por mayor y menor, hoteles y restaurantes y servicios comunales, sociales y personales junto a la industria manufacturera.

La población con problemas de empleo mantiene un ritmo de crecimiento acelerado durante los últimos cinco años, principalmente los subempleados invisibles del área rural, recayendo la mayor incidencia de los ajustes al salario mínimo en el área urbana. En lo que respecta a los subempleos visibles, muestran un ritmo de crecimiento más moderado, soportando la principal incidencia de los ajustes al salario mínimo la población del área rural en edad joven, desempeñándose en las actividades de la agricultura, silvicultura, caza y pesca, comercio por mayor y menor, hoteles y restaurantes y servicios comunales, sociales y personales junto a la industria manufacturera. Este comportamiento se debe en gran medida a la falta de políticas de protección social, en donde existe un limitado número de trabajadores amparado por algún tipo de asistencia social. 
La población asalariada en su totalidad muestra un crecimiento moderado respaldado en gran medida por el sector público y privado, en donde los asalariados públicos radican en las actividades de servicios comunales, sociales y personales, electricidad, gas y agua y transporte, almacenamiento y comunicaciones, dependientes de la población con nivel de educación secundaria y superior. En cambio, los privados dependen en su mayoría de las actividades de la agricultura, silvicultura, caza y pesca, la industria manufacturera y el comercio al por mayor y menor, hoteles y restaurantes, dependiendo principalmente de la población con nivel de educación primaria y secundaria del área urbana principalmente.

\section{BIBLIOGRAFÍA}

Comisión Económica para América Latina y el Caribe. (1997). Reflexiones sobre los indicadores del mercado de trabajo para el diseño de políticas con un enfoque basado en el género. Santiago de Chile.

Consejo Hondureño de la Empresa Privada. (2012). Acuerdo salarial en Honduras. Memoria comentada sector privado. Tegucigalpa.

Del Cid, J. R. (2011). Impacto de los reajustes al salario mínimo en Honduras. Tegucigalpa.

Instituto Nacional de Estadística. (2011). Encuesta permanente de hogares de propósitos múltiples. Tegucigalpa.

Javeriana, P. U. (2003). Empleo y desempleo en Colombia: el impacto de la legislación laboral y de las políticas salariales (1976-1999). Bogotá.

Organización Internacional del Trabajo. (2010). Informe mundial sobre salarios 2010/2011. Santiago de Chile.

Organización Internacional del Trabajo. (1999). Definiciones internacionales y futuro de las estadísticas del subempleo. Suiza.

Organización Internacional del Trabajo. (2012). Análisis diagnóstico del empleo. Suiza.

Secretaría de Trabajo y Seguridad Social. (2010). Estudio del mercado de trabajo 2010-2011. Tegucigalpa.

Secretaría de Trabajo y Seguridad Social. (25 de agosto de 2012). Recuperado de: http://www.trabajo.gob.hn/organizacion/dgt-1/direccion-general-desalarios/decretos 